\title{
Algunos problemas de fundamentación y justificación sociológica: Apuntes en el marco del debate teórico de la sociología contemporánea
}

\author{
Some problems typically present in sociological justification and foundation: Notes on the theoretical \\ debate regarding contemporary sociology
}

\section{Maynor Antonio Mora}

Universidad Nacional, Costa Rica, mmoraa90@hotmail.com

doi: http://dx.doi.org/10.15359/abra.35-50.1

\begin{abstract}
Resumen: En este ensayo se tratan algunos problemas propios de la fundamentación y justificación sociológica contemporánea y sus implicaciones desde una postura epistemológica construccionista, pragmática y evolutiva. Como tesis central se plantea la necesidad de retomar arqueológicamente (en el sentido planteado por Foucault, es decir, desde la reconstrucción de las capas profundas de nuestra propia contemporaneidad) dichos problemas (desde el origen de la disciplina), sin obviar sus derroteros y el bagaje histórico que la misma ha producido dentro del marco de la modernidad en el último siglo y medio de desarrollo histórico, con el fin de evadir la imposición renovada del sentido común, el sentido común sociológico y la ideología, camuflados en muchos casos bajo la técnica o una utopística acrítica.
\end{abstract}

Palabras clave: Sociología, fundamentación epistemológica, justificación epistemológica, construccionismo social, pragmatismo, evolucionismo.

\begin{abstract}
In this paper some problems typically observed in contemporary sociological justification and foundation, as well as its implications, are discussed from a constructivist epistemological, pragmatic and evolutionary position. The central thesis proposed here is the need to reconsider archaeologically these problems (from the origin of the discipline and in the sense proposed by Foucault, i.e. from the reconstruction of the deep layers of our own contemporaneity). These problems should be reconsidered without forgetting the course of sociology and its historical background in modern times, specifically in the last century and a half of its historical development, in order to avoid the renewed imposition of common sense, sociological common sense and ideology, which in many cases are camouflaged under the technique or uncritical utopistics.
\end{abstract}

Keywords: Sociology, epistemological foundation, epistemological justification, social constructionism, pragmatism, evolutionism.

Fecha de recepción: 28-07-2014 Fechas de reenvío: 14-10-2014/20-10-2014/24-11-2014/26-01-2015/12-02-2015 Fecha de aceptación: 12-02-2015 Fecha de publicación: 11-04-15

Maynor Antonio Mora

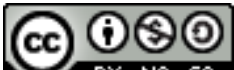

Esta obra está bajo licencia

Creative Commons Reconocimiento-NoComercial-Compartirlgual 4.0 Internacional License 
Después de un recorrido tortuoso, accidentado, conflictivo, sujeto a malos entendidos, pero rico y productivo, de la sociología que ha girado alrededor del debate casi permanente sobre su cuestionado carácter científico y, con mucho menos énfasis e interés, sobre su papel tecnológico (Lamo, 2011, p. 44), en donde resulta de lleno aventurado buscar una respuesta certera o única al problema.

Por lo tanto, es necesario retomar, contemporizadamente algunas de las cuestiones que han dado fundamento y justificación epistémicas a la sociología. Esta reconstrucción parcial que se plantea no debe malinterpretarse como resultado de una debilidad, o bien, como retorno al origen perdido, sino como una reconceptualización, idea que se asocia a la perspectiva construccionista, en el sentido de que la realidad es socialmente construida por y desde los agentes sociales; y al enfoque pragmatista que vincula el criterio de veracidad de los saberes sociales y del mismo conocimiento científico con su efectividad tecnológica, y así solventar indirecta pero, a su vez, ontológicamente, una salida respecto del relativismo .Aunque en algunos casos, construccionismo se asocia al constructivismo o, en el caso de obras en otros idiomas, se usa indistintamente, aquí mantendremos este definiens del concepto en cuestión, pese a la posible variabilidad del definiendum. Por lo que es necesario reconocer primero los siguientes dos eventos históricos concurrentes en la actualidad:

a) La excesiva positivización esencialista de la sociología, la cual desoye muchas veces sus fundamentos de ciencia que busca conocer lo social.

b) Su contaminación evidente con efectos técnicos (muchos de ellos instrumentales; además de poder diferenciar entre técnica y tecnología, esta última se entiende como saber epistemológicamente constituido) y utopísticos poco claros que la han decantado como sociotécnica (intentos no criticados ni evaluados que buscan transformar acríticamente la realidad social) y, en el peor de los casos, como ideología (en el sentido, por ejemplo, marxiano del término).

El acto de retomar los fundamentos, lo entendemos, por ende, asociado a una interpretación arqueológica en el sentido planteado por Foucault (2003) y la Hermenéutica en general. No se quiere caer ni en el esencialismo extremo propio del evolucionismo progresista y realista ni en el relativismo; por lo que, los argumentos que siguen oscilan entre un evolucionismo débil mnésicamente complejo, organizado y diversamente operativo (mas no progresista) mediado por el pragmatismo (no instrumental) del saber y el construccionismo (no relativista, pero sí comunicativo, intercultural y decolonial). A este enfoque lo podemos denominar construccionismo pragmático evolutivo (Pacho, 2009, sobre la epistemología evolucionista; Corcuff, 2005, sobre el construccionismo estructuralista en Bourdieu; Corcuff, 2009, respecto del construccionismo en Berger y Luckmann; Kratochwil, 2013; Porta y Keating, 2013, sobre el denominado pluralismo pragmático).

Gran parte de la sociología se ha institucionalizado dentro de la cultura occidental y sus principales marcos de influencia dentro de un proceso de globalización imperial, unidimensional (Marcuse, 1969), destructor de la otredad (Appadurai, 2007 Honneth,2011); pese a la integración objetiva de esta misma globalización (o sistema mundo, según lo plantean autores 
como Wallerstein en su diversa obra), que no podría haberse constituido sin el aporte de la racionalidad científica, la técnica y la tecnología, las ciencias sociales y las sociotécnicas (Latour, 1998, 2007; Callon, 1998), independientemente de la existencia de enfoques críticos y/o alternativos.

El mundo moderno está vinculado a una espacialidad o zona de la realidad afectada por el tiempo imaginario individual/colectivo, referido aquí específicamente a la modernidad occidental, no occidental y sincrética (Beriain, 2005; Latour, 2007) e incluso analéptica como ha propuesto, en general, la filosofía y la teología de la liberación en su modo de ser estrictamente latinoamericano o africano. La historia jerárquica y genéricamente comprendería diversas culturas; estas, diversas intraculturas (en permanente diálogo) y, dentro de estas se encontrarían paradigmas e influencias complejas, tanto dominantes como no dominantes, que es necesario discernir sin que esto implique un proceso de taxonomización social, sino, más bien vera la cultura como un río con sus flujos y reflujos en permanente cambio y transformación donde es necesario la descripción, tanto para la explicación y la aplicación del pensamiento crítico, como para que facilite los respectivos procesos de decantación política y epistemológica.

El paradigma se utiliza aquí en sentido amplio, ligado a las formas de vida y no exclusivamente a los sistemas teóricos propiamente filosófico/científicos. No se puede establecer una relación comprehensiva entre cultura, subcultura y paradigma. E igualmente, los mundos culturales no occidentales, desde una postura intercultural y decolonial estricta, no deben (ni en realidad pueden) ser evaluados mediante categorías occidentales ni modernas en particular, salvo los propios criterios de las distintas culturas y los aspectos comunes entre las mismas (supuesto que define en sentido estricto el enfoque intercultural). Desde una visión multinivel/plural/diversa, cultura(s), intracultura(s) y paradigma(s) se implican de forma compleja, sincrética, diacrónica, transespacial, e iteracional (como parte de un proceso de construcción histórica). A esto último lo podemos definir como un criterio evolucionista débil (no progresista).

Vale recurrir a la ingenuidad como estrategia de abordaje. No porque se pueda seguir en la ingenuidad, luego de más de un siglo y medio de aportes de la sociología, sino, porque en el camino la sociología se ha contaminado con los residuos propios de su fundamentación y desarrollo, y se ha confundido con la técnica (racionalización instrumental) que le es propia (sociotécnica), o que deriva de ella, es decir, ha caído en un pragmatismo acrítico (A:1; en el anexo se presenta una ampliación de segundo nivel que da soporte a las afirmaciones del ensayo; se sugiere la lectura del mismo en el caso de que se quiera profundizar en el tema; el numeral en cada caso corresponde al enunciado respectivo en dicho anexo).

Una evaluación crítica supone eliminación de las viejas asociaciones positivistas que giran alrededor del término pragmatismo, puesto que lo vinculan con un quehacer social estrictamente individualista y economoproductivista (A: 2). Más bien, por pragmatismo evolutivo, se busca entender, al seguir de alguna manera la crítica marxista, que el quehacer de la sociología está sujeto a su carácter praxeológico, socialmente crítico; pero, a fin de cuentas, vinculado con la práctica de las y los sujetos individuales y sociales en su quehacer. No se usa en general el término praxeológico, en el sentido de que el término pragmático es mucho más amplio, una vez liberado de su carga individualista, y de su viejo

Maynor Antonio Mora 
raigambre con el liberalismo posesivo, donde ha tenido hasta ahora resultados muy limitados sociológicamente, a la vez que desde un punto de vista político muy negativos.

La reflexión por realizar seguirá los pasos de lo que se denomina metateoría o reflexión de segundo orden (en algunos casos filosófica) sobre las posibilidades teóricas de la sociología, misma que parece carecer del grado de seguridad de las ciencias de la naturaleza, lo cual plantea una distancia inexistente entre ambas formas de conocimiento (Latour, 2007; Domènech y Tirado, 1998).

No se cae en este lugar por lo común del mismo, sino por la innegable sospecha que despierta la afirmación, tantas veces enunciada en las últimas décadas, sobre las supuestas consecuencias de que la sociología se construya desde una condición multiparadigmática, esto es, teóricamente heterogénea $y$, en algunos casos, ambivalente, sujeta a una permanente arqueología teórica (Foucault, 2003), como si las demás ciencias no requirieran de tal arqueología. Por lo que es necesario establecer líneas de reflexión, sobre tal particular, sin colaborar en el acto con la misma dispersión teórica, es decir, reconocer el carácter en parte constructivo y pragmático de la sociología y su objeto; pero, también, la necesidad de la crítica propositiva sobre ciertos conceptos acuñados en su origen y desarrollo como ciencia social, en tanto síntesis evolutiva.

La dispersión puede ser achacada a priori a:

a) Subjetivización extrema (no reconocida del todo) de los cánones de la investigación y la formulación de hipótesis o sistemas teóricos y explicativos.

b) Abrupta separación entre manejo de los sistemas teóricos y los conjuntos de datos de referencia.

c) Pronunciada confusión entre ciencia e ideología, y entre técnica y tecnología.

d) Un retorno racionalmente descontrolado a la filosofía social.

No se comparte el supuesto acerca de que la relación sociedad/sociología constituya una sociedad reflexiva (Lamo, 2011); cosa que solo puede definirse (en parte) desde el construccionismo epistemológico, es decir, aquel paradigma que sostiene que tanto las teorías como las formas de ver el mundo se van construyendo según las particularidades históricas y las relaciones entre los agentes y su inserción pragmática (epistemológicamente evaluada) dentro del mundo objetivo/natural, incluidos las/los científicos (pragmatismo pluralista en el sentido que queremos especificar). Los problemas que siguen tienen que ver con las aristas de esta situación general enunciada en la presente introducción.

La exposición comprende el debate según los elementos arqueológicos que se señalan a continuación. Los problemas analizados en sentido arqueológico son los siguientes: el fundacional, el de la objetividad, el de la diversidad de enfoques teóricos, el de la utopística, el de la tecnología, el de los efectos residuales, el de la socialización científica; y, a modo de conclusión, el problema de la memoria gnoseológica. 


\section{El problema fundacional}

La fundación de la sociología fue tardío, en referencia a las otras ciencias naturales y sociales, ya que en el caso de estas, amplias secciones de su objeto formaban parte de los sistemas teóricos, técnicos y tecnológicos, es decir, de formas de control/mediación con la naturaleza objetiva, aunque había elementos que individualmente pueden ser denominados como sociológicos, tanto en la cultura occidental, como en la cultura árabe y china (Graham, 2005). Este hecho no significa que la caverna (Platón), el sentido común, las prenociones, los idola (Bacon), la ideología (Marx), la imaginación pura, la doxa, no permearan desde tiempos inmemoriales el saber humano sobre/o más bien respecto del mundo objetivo/natural en general, así como relacionado con los mundos social y psíquico. La constitución del objeto arrastra un vicio extremadamente esencialista dentro de los principales conceptos de lo social: sujeto, individuo, persona, acción, sistema, sociedad, historia, lenguaje, etcétera.

La sociología se confunde con formas de normalización modernas y modernizantes, altamente cargadas por estas conceptualizaciones propias de la filosofía social; y, ante todo, por un vacío conceptual claramente justificable por sus propias condiciones de producción, al determinar la naturaleza del fenómeno social moderno, lejos de explicaciones como la lejanía o cercanía del investigador (sujeto, $=\mathbf{s}$ ) respecto del objeto, = $\mathbf{0}$; caso del pensamiento weberiano (Giner, 2012), el cual asume la distinción entre ciencias nomotéticas (naturales) e ideográficas (del espíritu o históricas). Parece de sumo valor la sociología simétrica al señalar la existencia de cuasiobjetos y cuasisujetos que son, a la vez, entes naturales, sociales y lingüísticos (Latour, 2007).

El instituto sociológico tiene un criterio de veracidad de alcance, al menos, semiuniversal (a esto lo denominamos criterio o principio evolutivo débil: A: 2); ya que, con independencia del contexto de fundación, no puede ser que este responda a unas condiciones de tan alta variabilidad que resulte objetivamente relativo, específico y único, lo cual sería simultáneo al postulado de una identidad redonda de la modernidad occidental, que negaría la trascendentalidad pragmático/intercultural de la sociología, al reducir su papel a una etnodescripción autorreferente, recursiva, redonda, cerrada y atemporal de la modernidad ( $\mathbf{A}$ : 3).

\section{El problema de la objetividad}

La objetividad de la sociología remite a dos subproblemas inscritos también en el proceso de fundación de las ciencias sociales. Estos son los subproblemas de la continuidad y la discontinuidad óntica. La continuidad refiere a lo siguiente:

a) Uniformidad relativa intrínseca del mundo objetivo natural.

b) Reducción de fragmentos del mundo objetivo natural a las mismas condiciones de determinación causal.

Maynor Antonio Mora

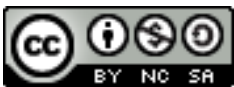

Esta obra está bajo licencia

Creative Commons Reconocimiento-NoComercial-Compartirlgual 4.0 Internacional License 
c) Semejanzas (homologías) en diversas dimensiones y planos del mundo objetivo natural.

d) Reducción del mundo objetivo natural a los mismos principios microscópicos y macroscópicos.

e) Interacción causal entre todas las zonas del mundo objetivo natural, en donde se incluye la realidad socialmente construida y el mundo psíquico (esta diferenciación del mundo en los tres mundos proviene ante todo de la teoría habermasiana (Habermas, 1999, 2007).

Desde las escalas micro hasta las macrofísicas, dicha continuidad es la que permite el establecimiento de leyes o postulados científicos de alto alcance de veracidad, según los principales enfoques epistemológicos (representacionales, verificacionistas, falsacionistas, construccionistas, performativos y pragmatistas).

La discontinuidad opera como un criterio epistémico de diferenciación entre unos pocos principios científicamente concluyentes (A: 4) y las observaciones concretas (casi todas concentradas en la modernidad centro/occidental de los últimos 150 años). Cada manifestación social, aparece, como lo señala Durkheim (1980) en el siglo XIX, de manera sui generis. Esta ausencia refrenda, hasta cierto punto, la definición de la sociología como ciencia de la modernidad en las siguientes dos acepciones:

a) Ciencia que estudia la modernidad.

b) Ciencia que legitima (sociotécnica e ideológicamente) la modernidad.

Esta especificidad (un objeto general o cultural diferenciado, a efectos de evitar el lexema civilización) genera una relativa fisura epistémica, con consecuencias en la constatación de regularidades objetivas (A: 5).

La relación de conocimiento $(\mathbf{=} \mathbf{c})$ opera en las ciencias sociales como en todas las demás; en la forma $\mathbf{c}(\mathbf{s} \rightarrow \mathbf{0})$ (conocimiento, como relación de un sujeto=s, respecto de un objeto $=$ $\mathbf{o}$ ). Los paréntesis en $(\mathbf{s} \rightarrow \mathbf{0})$ indican, primitivamente, la ruptura óntica, mediante la condición hiancial introducida por el conocimiento, bajo la forma no positiva de c (conocimiento) sino negativa como (...). c, siempre va a suponer $\mathbf{s}^{\prime} \rightarrow \mathbf{0}^{\prime}$, una relación entre un sujeto $\left(\mathbf{s}^{\prime}\right)$ y un objeto ( $\left.\mathbf{o}^{\prime}\right)$ de segundo orden. A lo que se agrega la introducción de un segundo momento de $\mathbf{c}, \mathbf{c}^{\prime}$, bajo la forma $\mathbf{c}^{\prime}(\mathbf{s} \rightarrow \mathbf{0})$ que retrotrae el proceso de conocimiento bajo una espiral con tendencia a la infinitud definido como (...).

Los contenidos $\mathbf{c}^{\prime}(\mathbf{x}), \mathbf{c}^{\prime \prime}(\mathbf{y}), \mathbf{c}^{\prime \prime \prime}(\mathbf{z})$ implican movimientos ónticos que suponen no solo la evolución (en el sentido débil que defendemos) del conocimiento, sino la transformación relacional de s, o y c, siempre hacia (...). La ruptura negativa (...) indica la condición reflexiva y no casuística de la fundamentación del conocimiento, a diferencia del saber en general.

El principio relativo de discontinuidad óntica del objeto parece, por ahora, un escollo difícil de superar, con peligros objetivos, como la sobrecarga técnico/instrumental. Dicho principio encuentra salida en la comparación histórica de sucesos sociales. Por consiguiente, 
agréguese a la premisa de Durkheim, que la ciencia no funciona solamente mediante hipótesis contrastativas, verificativas, refutativas, o bien representacionales teoría/realidad; sino también constructivas y prácticas sujetas a una racionalidad hermenéutica y arqueológica críticamente acumulativa en el caso de lo social (construccionismo pragmático evolutivo).

La utopística epistémica funciona en sentido negativo como marco de argumentación de instrucciones técnicas y/o tecnológicas. La comunicación de la sociología con las ciencias de la naturaleza (lo que Bruno Latour, 1998, 2007 define como sociología simétrica o sociología de la red conocimiento-sujeto-tecnología-naturaleza objetiva; Gabriel Tarde sugirió de otra forma desde finales del siglo XIX; y, en especial, Gregory Bateson, 1993 ha afirmado, en relación con la constelación de las ciencias) puede saldar esta falla inicial de la continuidad epistémica.

\section{El problema de la diversidad de enfoques teóricos}

La sociología se manifestó desde un inicio desde diversas posturas teóricas. Tal situación podría achacarse al vínculo entre tales ciencias y la filosofía social, donde corrientes y/o sistemas teóricas/os se presentan opuestos, yuxtapuestos o superpuestos, o bien a la complejidad de lo social.

Un principio evolucionista débil puede observar lo real/social desde diferentes puntos de vista, separados por saltos ónticos, históricos y epistémicos, con posibles detenciones y reversiones. Se sostiene aquí esta última tesis respecto de la evolución del conocimiento sociológico, sujeta no solo a condiciones ónticas trascendentales del mundo objetivo natural en general, sino también a los procesos sociales (A: $4,5,6)$.

La orientación sociocognitiva resuelve, en alguna medida, la aparente petición de principio inherente a la idea de la cercanía del objeto, lo cual destruye las oposiciones clásicas, aparentemente irreductibles, naturaleza/cultura, teoría/práctica, idealismo/materialismo, sujeto/objeto, solipsismo/pluralismo de las mentes (Giner, 2012).

\section{El problema de la utopística}

Este no solo incluye a la sociología, sino a todas las ciencias, a través de las dos formas mediante las que se ligan con la ética (Blackburn, 2006): activa y pasiva. La forma pasiva deviene del problema de la ética científica, así como de los criterios de normatividad epistémica (Broncano y Vega, 2009). Las ciencias y, en especial, las ciencias de la naturaleza mantienen una especie de consenso tácito sobre lo que no debe hacer la investigación, en especial mediante la bioética.

La bioética es una de las contracaras de la ciencia, especialmente en sus manifestaciones más positivas (que argumentan una función cognoscitiva estrictamente instrumental del conocimiento), las cuales no previeron originalmente, que todo acto de este involucra la vida como condición de posibilidad (Ellacuría, 1990; Dussel, 1998).

Maynor Antonio Mora

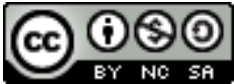

Esta obra está bajo licencia 
Las ciencias naturales, que aparecen como ciencias en sí y nada más, no son respaldadas siempre por una verdadera observación de su contexto epistémico global, vinculado con la condición social del evento humano. Aunque validen sus efectos desde el punto de vista ético, solo lo validan en sentido estricto de ciencias de la naturaleza (del mundo objetivo natural), al igual que las ciencias sociales, también son ciencias de la naturaleza de segundo orden. En el caso de las ciencias sociales, el desarrollo bioético ha sido neutralizado como parte de una positivización empírica; o se ha decantado hacia la orientación utopística activa sin mayor dilucidación desde el punto de vista pasivo $(\mathbf{A : ~} \mathbf{7}, \mathbf{8})$.

\section{El problema de la tecnología}

Durante los siglos XVIII y XIX, la economía distingue entre lo analítico y lo normativo; el resto de ciencias sociales, en mayor o menor medida, nacieron también como ciencias normativas, interesadas en la transformación de su objeto (una violenta conversión de la sociedad tradicional europea en sociedad moderna). Las implicaciones sociotécnicas en el caso de la sociología han sido estudiadas, pero quizás no lo suficiente, porque en algunos casos no se ha asumido abiertamente la existencia de efectos técnicos de esta (Lamo, 2011), más allá de las funciones ético-políticas estrictamente normativas.

En el caso de las ciencias de la naturaleza objetiva, tales funciones, sí han caído bajo el término directamente alusivo de tecnología (en realidad técnica), y no de simple normatividad funcional, salvo funciones que han sobrecaído epistemológica y axiológicamente debajo del análisis bioético indirecto derivado, pero de manera muy cercana a subobjetos de las ciencias humanas, entre ellas la medicina. $O$, porque las funciones técnicas (instrumentales) refieren a criterios de utopística pasiva o funciones ético-políticas normativas expresamente declaradas, sin margen residual abiertamente reconocido como efectos realidad puros, o efectos consciencia (A: 9).

Las sociedades modernas se han recargado de transformaciones, estructuras, prácticas y sistemas técnico/instrumentales (A: 10), en donde se generan procesos ético-políticos de normalización/desnormalización, más allá de todo cálculo del riesgo, tanto en el marco de una utopística negativa (incapaz de reconocer los eventos técnicos) como de una utopística positiva (aquella que supone efectos esperados, pero que niega o no visualiza el conocimiento de eventos residuales fuera de sí y del marco técnico).

\section{El problema de los efectos residuales}

Se denomina aquí efectos residuales, ónticos de segundo orden o efectos objetivos socionaturales a aquellos que derivan, directa o indirectamente, del conocimiento de la sociología en particular y de las ciencias en general. Se incluye la acumulación de patrones de comportamiento agencial social y socioecológicos, surgimiento de nuevos patrones, transformación de los mismos sistemas o cambio en las relaciones del comportamiento agencial o de las sociedades, tanto en el marco del tiempo entrópico como del imaginario (y sus imbricaciones): se incluye cualquier relación causa/efecto, donde el efecto deriva del mismo evento del conocimiento como evento real (A: 11). 
Este principio respalda toda epistemología construccionista (Corcuff, 2005; Porta y Keating, 2013), aunque puedan surgir diferencias a título particular de los diversos debates epistemológicos respecto del avance del neopragmatismo y/o la teoría crítica en los últimos treinta años. Todo acto de conocimiento es evento real: está encadenado a la totalidad de eventos natural/objetivos y, por ende, está sujeto a relaciones de causalidad y demás relaciones epistémicas (A: 12).

La utopística epistémica (antiutopística, la distopística y la heterotopística), en su sentido activo, se asume, no obstante, como lo siguiente:

a) Acto normativo trascendental de conocimiento.

b) Acto carente de residuo, del cual no se desprende ninguna consecuencia otra, más allá de las consecuencias esperadas.

El desarrollo científico ha sido relativamente anárquico, respecto a la descripción, explicación, representación y/o comprensión de dichos efectos residuales, muchos paradójicos, especialmente si se relacionan con la orientación técnica (instrumental) ya descrita, sea en su versión consciente o inconsciente, tanto para las condiciones de vida humana como de la vida en general dentro del planeta (A: 13).

La sociología de los efectos residuales de la sociología no ha tenido un suficiente desarrollo como subdisciplina, salvo los enunciados certeros de Elias (1990) y Bourdieu (2000); que no dieron pie a una amplia y sólida continuidad, excepto la actividad de algunos investigadores (destaca el trabajo de Bruno Latour y otros autores), al haber una excesiva insistencia (no siempre consciente) en la disyunción de las partes pasiva y activa de la utopística: ansiedad cientificista por abarcar conocimiento de lo social, por un lado, e impulso por transformarlo por otro (prerrogativa de una racionalidad desgajada, podría decirse según Habermas (1999 y 2007) (A: 14).

\section{El problema de la socialización científica}

Al respecto del problema de la socialización científica es necesario partir de una serie de supuestos relacionados con dicha situación:

a) La ciencia moderna, desde un comienzo, se ha pensado como un sistema demográficamente limitado. Dicha limitación supone inclusiones y exclusiones en la construcción del conocimiento, esto es, un complejo saber/poder (Foucault, 2008).

b) La diferencia entre expertise y socialización científica de la población ha sido accidentada e irregular.

c) Fuera del marco de la expertise ha habido una relativa generalización del imaginario científico mediante el aprendizaje social de los productos de conocimiento (saber científico) y, en menor medida, de las reglas básicas de funcionamiento de los métodos científicos; así

Maynor Antonio Mora

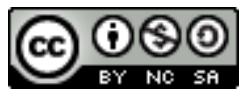

Esta obra está bajo licencia 
como de la preexistencia obvia de marcos pre o paracientíficos en todo nivel de la realidad socio/natural (esto, hay que insistir, dentro y fuera de la cultura moderna).

d) La educación formal ha asumido relativamente el carácter científico del conocimiento. Lo anterior ha calado en la relativa secularización de la sociedad moderna como sociedad asociada a la ciencia (Lamo, 2011), desde un principio progresista del sentido común científico y del sentido común sociológico (Bourdieu, 2000 y 2005).

e) El sentido común científico, no garantiza, en forma alguna, una generalización de la ciencia, sino solo de sus productos menos dificultosos (saber científico), de la ideología científica y de la técnica (saber instrumental).

f) La generalización de la ciencia resulta dificultosa: supone problematizar la idea de una sociedad puramente científica, pensada desde el punto de vista de algunas hipótesis extremas provenientes del sentido común producido por las ciencias naturales, caso de la cosmología (Tipler, 1997) o de las neurociencias. El alto grado de diferenciación y especialización de la ciencia ni siquiera permite tener nada parecido a una tal consciencia, dada la forma abrupta y cognoscitivamente discontinua en que evoluciona la misma. Los productos de conocimiento tienden a ser altamente diversos y amplios, y probablemente inmanejables para una agencia unitaria, fuera de las mismas generalizaciones metateóricas y del saber institucionalizado como sentido común. La ciencia evoluciona desperdigando efectos real/objetivos, que impactan las formas de interpretación y de acción social, sin que estas tengan alguna connotación que implique una estricta socialización científica.

Esto supone una clara violencia epistémica de la ciencia contra dichos saberes (incluye formas altamente complejas de conocimiento), y pese a la alta justificabilidad y razonabilidad de la ciencia (idea de Habermas y otros autores que defienden con gran sinceridad el potencial racional de la modernidad como herencia de Occidente a la humanidad), y la preexistencia de formas pre o paracientíficas propias de todas las culturas y de sus respectivos mundos de la vida (idea de fondo de la decolonización epistémica). En el caso de la sociología y las ciencias sociales, aparte de su obvia especialización y diferenciación, se han empantanado y confundido con sus efectos real/objetivos y con las sociotécnicas, lo cual rebaja su horizonte epistémico. Esto reclama un inevitable y eterno retorno subtrascendental y dialéctico de la reflexión sobre sí misma, respecto de cualquier acabamiento de la ciencia o de la historia

Cuando Bourdieu (2000) critica, en los años 60 del siglo pasado, el avance del sentido común sociológico, piensa en una sociología altamente especializada, que debe garantizar seguridad epistémica sobre su propio proceso de autoconstitución. Se trata de lo que él y otros autores reconocen como vigilancia epistemológica; siempre en el marco de una sociología reflexiva (Bourdieu y Wacquant, 2005). La vigilancia epistemológica supone una proyección infinita bajo la forma: $\mathbf{c}\left(\mathbf{c}^{\prime \prime}\left(\mathbf{c}^{\prime}(\mathbf{c}(\mathbf{0} \rightarrow \mathbf{s}))^{\prime}\right)^{\prime \prime}\right) \infty, 0(\ldots)$.

El daño de tal proyección supone una ruptura de la evolución epistémica (comunicabilidad histórica del conocimiento), lo que deriva en fallas epistémicas de todo tipo, que incluye 
recursividades del sentido común, efectos objetivos, efectos técnicos no previstos y eventos riesgo (A: 15)

\section{Conclusión: el problema de la memoria gnoseológica}

Por ella se entiende, finalmente, la institucionalización sociocognitiva de la sociología, dentro del marco espaciotemporal entrópico e imaginario (Bagú, 1973; Beriain, 1997; Elias, 1989), es decir, como parte de la realidad objetiva y de la cultura. Aquí es posible ver el problema hacia el pasado mnésico (como carga de datos operacionales de baja entropía) y hacia el tiempo imaginario inherente a la dinamización de los datos operacionales mediante todos los sistemas organizativo/institucionales y las agencias sociales mediante la cognición individual/colectiva y sus referentes culturales -lo que diversos autores, entre ellos Niklas Luhmann, denominan sistema(Luhmann, 1990).

La memoria aparece como conjunto estructurado de directrices agenciales enmarcado en el presente operativo real/social/cultural; por un lado surge como necesidad de efectuar nuevos procesos de conocimiento de los efectos realidad, en tanto no quedan evidentes (ni tienen por qué) en la proyección del futuro entrópico, mediante el tiempo imaginario. Por otro lado, se concibe aquí lo cognitivo no solo en un sentido individual, sino en un sentido socio/cultural, que supone la relación entre la constitución cognitiva del individuo y los sistemas culturales que referencian todo proceso de constitución individual. El conocimiento sociológico se define como variable espaciotemporal entrópica y como parte de un complejo multidimensional.

Por lo tanto, resulta necesario ampliar el proceso de investigación sociológica desde el punto de vista tanto arqueológico (en el sentido foucaultiano de una crítica propositiva de las bases mismas de la sociología) como en un sentido constructivo-pragmático; en especial, del tiempo imaginario individual/cultural, así como de las múltiples orientaciones de la acción, de los movimientos y decisiones de las agencias constituidas fuera de las tres grandes figuras clásicas de la acción sociocognitiva propuestas por la filosofía y la fenomenología de la consciencia/razón/voluntad (sujeto/individuo/persona) hasta ahora dominante en el marco de los tiempos entrópico y sociocognitivo.

Estas y otras categorías de la sociología pueden, en mayor o menor medida, revisarse a la luz de esta evidente multidimensionalidad socionatural, así como de los supuestos que han soportado los problemas hasta aquí reconocidos. Lo que permite continuar el debate que coadyuve a restringir, una vez más, el agresivo y permanente retorno del sentido común y de la utopística pura y voluntarista no atravesada por un proceso de análisis epistemológico crítico de sus efectos.

\section{Referencias}

Appadurai, A. (2007). El rechazo de las minorías Ensayo sobre la geografía de la furia. Barcelona: Tusquets Editores, S. A. 
Arce, J. L. (2007). Teoría del conocimiento. Madrid: Editorial Síntesis, S. A. Recuperado de http://www.sintesis.com/themata-83/teoria-del-conocimiento-ebook-1410.html

Bagú, S. (1973). Tiempo, realidad social y conocimiento Propuesta de interpretación (2 ${ }^{\text {da }}$ ed.) México, D. F.: Siglo XXI Editores, S. A. Recuperado de https://books.google.co.cr/books?id=qL7lh6BKoy4C\&pg=PP6\&lpg=PP6\&dq=Editores + siglo+XXI+M\%C3\%A9xico+Tiempo,+realidad+social+y+conocimiento+Propuesta+de+i nterpretaci\%C3\%B3n,\&source=bl\&ots=aRAgbcVRI \&sig=3re8nWBYVUNa7V2SHXuR fcjXeSQ\&hl=es\&sa=X\&ei $=$ A3IVOGRJZDksATpnIDoBQ\&redir esc $=\mathrm{y} \# \mathrm{v}=$ onepage $\& \mathrm{q}=$ Editores\%20siglo\%20XX1\%20M\%C3\%A9xico\%20Tiempo\%2C\%20realidad\%20social \%20y\%20conocimiento\%20Propuesta\%20de\%20interpretaci\%C3\%B3n\%2C\&f=false

Bateson, G. (1993) Una unidad sagrada. Barcelona: Gedisa. Recuperado de http://www.casadellibro.com/libro-una-unidad-sagrada-pasos-ulteriores-hacia-unaecologia-de-la-men-te/9788474324808/33356

Beriain, J. (2005). Modernidades en Disputa. Barcelona: Anthropos Editorial. Recuperado de http://www.anthropos-editorial.com/DETALLE/MODERNIDADES-EN-DISPUTA-ATTCS$\underline{042}$

Blackburn, P. (2006). La ética Fundamentos y problemas contemporáneos. México, D. F.: Fondo de Cultura Económica. Recuperado de http://www.fondodeculturaeconomica.com/Librerias/Detalle.aspx?ctit=040085R

Bourdieu, P. (2000). El oficio del sociólogo. México, D. F.: Siglo XXI Editores, S. A. Recuperado de https://books.google.co.cr/books?id=7GV2ezilRgQC\&pg=PA6\&lpg=PA6\&dq=siglo+xxi +editores+s.+a+el+oficio+del+sociologo\&source=bl\&ots=XLVPjoO5gg\&sig=d9Don 4T ki829y8M91a-

H7yzdW4\&hl=es\&sa=X\&ei=d1zZVLPxAYKlgwT0xYKQCw\&redir esc=y\#v=onepage\&q =siglo\%20xxi\%20editores\%20s. \%20a\%20el\%20oficio\%20del\%20sociologo\&f=false

Bourdieu, P. y Wacquant, L. (2005). Una invitación a la sociología reflexiva. Buenos Aires, Argentina: Siglo XXI Editores S. A.

Broncano, F. y J. Vega (2009). Las fuentes de la normatividad epistémica: deberes, funciones, virtudes. En Quesada, D. (Ed.) Cuestiones de teoría del conocimiento. Madrid: Editorial Tecnos, S. A. Pp. 77-110. Recuperado de http://www.anaya.es/cgigeneral/newFichaProducto.pl?obrcod=2228102\&id sello editorial web=12\&id sello VisualizarDatos $=12$

Callon, M. (1998). El proceso de construcción de la sociedad. El estudio de la tecnología como herramienta para el análisis sociológico. En Domènech, M. y Tirado, $F$. J. (Eds.)Sociología simétrica Ensayos sobre ciencia, tecnología y sociedad. Barcelona: Editorial Gedisa. Pp. 143-70. Recuperado de 
http://www.gedisa.com/ficha.aspx?cod=302448\&titulo=Sociolog\%C3\%ADasim\%C3\%A9trica\#.VNlgCuaG8Yk

Corcuff, P. (2005). Las nuevas sociologías. Madrid: Alianza Editorial, S. A. Recuperado de http://www.sigloxxieditores.com.ar/fichaLibro.php?libro=978-987-629-264-1

Domènech, M. y F. J. Tirado (1998). Claves para la lectura de textos simétricos. En Domènech, M. y Tirado, F. J. (Eds.).Sociología simétrica Ensayos sobre ciencia, tecnología y sociedad. Barcelona: Editorial Gedisa. Pp. 13-50. Recuperado de http://www.gedisa.com/ficha.aspx?cod=302448\&titulo=Sociolog\%C3\%ADasim\%C3\%A9trica\#.VNlgCuaG8Yk

Durkheim, E. (1980). Las reglas del método sociológico. Buenos Aires: Editorial La Pléyade. Dussel, E. (1998). Ética de la liberación en la edad de la globalización y de la exclusión. Madrid: Editorial Trotta, S. A. Recuperado de http://www.trotta.es/pagina.php?cs id pagina $=13 \&$ cs id contenido=1555

Elias, N. (1989). Sobre el tiempo. México, D.F.: Fondo de Cultura Económica. Recuperado de https://www.fcede.es/site/es/libros/detalleslibro.asp?IDL=7173

Elias, N. (1990). Compromiso y distanciamiento. Barcelona: Ediciones Península. Recuperado de

http://books.google.co.cr/books/about/Compromiso y distanciamiento.html?id=1tE AAAA CAAJ\&redir esc $=\mathbf{y}$

Ellacuría, I. (1990). Filosofía de la realidad histórica. San Salvador, El Salvador: UCA editores. Recuperado de http://www.uca.edu.sv/filosofia/index.php?cat=10

Feyerabend, P. (1986). Tratado contra el método Esquema de una teoría anarquista del conocimiento. Madrid: Editorial Tecnos, S. A. Recuperado de http://books.google.co.cr/books/about/Tratado contra el m\%C3\%A9todo.html?id=RjhvrzjjO qlC\&redir esc $=y$

Foucault, M. (2003). La arqueología del saber (21 ${ }^{\mathrm{era}}$ ed.). México, D. F.: Siglo XXI Editores, S. A., de C. V.

Foucault, M. (2008). El orden del discurso (4 ${ }^{\text {ta }}$ ed.). Barcelona: Tusquets Editores. Recuperado de http://www.tusquetseditores.com/titulos/fabula-el-orden-del-discurso$\underline{\text { fabula }}$

Giménez, J. (2010). Entender mejor la libertad: Un enfoque interdisciplinar entre neurociencia y filosofía. En F. Rodríguez, J. Arana y C. Diosdado (Eds.). Neurofilosofía Perspectivas contemporáneas, pp. 177-190. Madrid: Plaza y Valdez, S. L. Recuperado de http://www.plazayvaldes.es/libro/neurofilosofia/1370/ 
Giner, S. (2012). Teoría sociológica clásica (3 ${ }^{\text {era }}$ ed.). Barcelona: Ariel. Recuperado de http://www.planetadelibros.com/teoria-sociologica-clasica-libro-50487.html

Graham, A. Ch. (2005). El Dao en disputa La argumentación filosófica en la China antigua. México, D. F.: Fondo de Cultura Económica. Recuperado de http://www.fondodeculturaeconomica.com/Librerias/Detalle.aspx?ctit=004356LE

Gutiérrez, G. (2000). Ética y decisión racional. Madrid: Editorial Síntesis, S. A. Recuperado de http://www.sintesis.com/hermeneia-81/etica-y-decision-racional-ebook-488.html

Habermas, J. (1999). Teoría de la Acción Comunicativa II Crítica de la razón funcionalista. Madrid: Grupo Santilla de Ediciones, S. A.

Habermas, J. (2007). Teoría de la Acción Comunicativa I Racionalidad de la acción y racionalización social. México, D. F.: Aguilar, Altea, Alfaguara, S. A., de C. V.

Honneth, A. (2011). La sociedad del desprecio. Madrid: Editorial Trotta, S. A. Recuperado de http://www.trotta.es/pagina.php?cs id pagina=13\&cs id contenido=33639

Iglesias, J. y Herrara, M. (2005). Teorías sociológicas de la acción. Madrid: Editorial Tecnos, S. A. Recuperado de http://www.casadellibro.com/libro-teorias-sociologicas-de-laaccion/9788430942312/1015915

Kratochwil, F. (2013).Construccionismo: qué (no) es y su importancia. En D. Porta y M. Keating (Eds.).Enfoques y metodologías de las ciencias sociales Una perspectiva pluralista, pp. 93-119. Madrid: Ediciones Akal, S. A. Recuperado de http://www.akal.com/libros/Enfoques-y-metodologlas-en-las-CienciasSociales/9788446030621

Lamo, E. (2011). Mundialización, posmodernidad, reflexividad. En S. Giner (ed.).Teoría sociológica moderna(2 ${ }^{\text {da }}$ ed.). Madrid: Ariel, S. A., pp. 27-50. Recuperado de http://ariel.claudator.com/libro/teoria-sociologica-moderna-978843441704

Latour, B. (1998). De la mediación técnica: sociología, filosofía, genealogía. En Domènech, M. y Tirado, F. J. (Eds.). Sociología simétrica Ensayos sobre ciencia, tecnología y sociedad. Barcelona: Editorial Gedisa. Pp. 249- 303. Recuperado de http://www.gedisa.com/ficha.aspx?cod=302448\&titulo=Sociolog\%C3\%ADasim\%C3\%A9trica\#.VNlgCuaG8Yk

Latour, B. (2007). Nunca fuimos modernos Ensayos de antropología simétrica. Buenos Aires: Siglo Veintiuno Argentina S. A. Recuperado de http://www.monoskop.org/images/f/fb/Latour Bruno Nunca fuimos modernos Ensayo de antropologia simetrica.pdf

Luhmann, N. (1990) Sociedad y sistema: la ambición de la teoría. Barcelona: Ediciones Paidós Ibérica, S. A. 
http://books.google.co.cr/books/about/Sociedad y sistema.html?id=fZQvaM 8 mAC\& redir $\mathrm{esc}=\mathrm{y}$

Marcuse, H. (1954). El hombre unidimensional Ensayo sobre la ideología de la sociedad industrial avanzada( $5^{\text {ta }}$ ed.). México, D. F.: Editorial Joaquín Mortiz, S. A. Recuperado de $\quad$ https://zoonpolitikonmx.files.wordpress.com/2013/10/marcuse-el-hombreunidimensional.pdf

Pacho. J. (2009). Epistemología evolucionista. Una epistemología naturalizada. En D. Quesada (Ed.).Cuestiones de teoría del conocimiento. Madrid: Editorial Tecnos, S. A.Pp. 314-349.

Porta, D. y Keating, M. (2013). Enfoques y metodologías de las ciencias sociales Una perspectiva pluralista. Madrid: Ediciones Akal, S. A. Recuperado dehttp://www.akal.com/libros/Enfoques-y-metodologlas-en-las-CienciasSociales/9788446030621

Tipler, F. J. (1997). La física de la inmortalidad Cosmología contemporánea: Dios y la resurrección de los muertos. Madrid: Alianza Editorial, S. A. Recuperado de: http://books.google.co.cr/books/about/La f\%C3\%ADsica de la inmortalidad.html?id= mOsILY1IPOQC\&redir esc $=y$

\section{Anexo}

\section{Aparato explicativo de segundo nivel}

1. Ingenuidad epistemológica equivale a simple cautela frente a las generalizaciones ontológicas desarraigadas de toda evaluación axiológica y epistemológica.

2. La sociología tiende a una generalización del contexto específico occidental, o falsa universalización socio/cultural moderna, por encima de la diversidad de todos los otros mundos sociales fuera de Occidente, mediante el recurso no reconocido de la sociología; y, con ello, el sacrificio del postulado de un principio evolutivo débil de la misma, esto es, que su surgimiento pudiese haber acaecido en otros mundos socio/culturales (sin caer bajo la rúbrica estricta de ciencia racional moderna y sin caer en un contrafáctico).

3. a sociología tiende a la defensa incuestionable del carácter restringido de la sociología a la cultura moderna, y con ella, a Occidente (ver Lamo, 2011).

4. El caso del evento humano reviste, las siguientes características que generan cierta ruptura de la continuidad objetivo natural: A) El evento humano es exclusivo, hasta donde se conoce, de la Tierra; por lo que carece de formas xenoculturales de comparación, contando solo con sus diversas manifestaciones históricas sui generis (Durkheim, 1980). B) Es evento relativamente corto (cubre algunos miles de años: 10 o 20) a diferencia de los demás sucesos reales, que tienen un umbral temporal amplio (millones o billones de años). C) Es 
explicado solo parcialmente por variables físicas, químicas, biológicas, etológicas, cognitivo/evolutivas y ecológicas. D) Expresa muy pocos aspectos de manera exclusivamente definitoria: a) gregarismo; b) transmisibilidad cultural; c) diferenciación/complejización relativas; d) expansión ecológica (hacia una relativa dominancia macro-ecológica); y, e) reproducción cultural adscrita de manera compleja a la reproducción macro-ecológica, mediante el lenguaje.

5. Cognoscitivamente pueden operarse reconstrucciones parciales de la discontinuidad en el marco temporal; pese a la salvedad que señala Foucault, en el sentido de que toda forma de decir el mundo supone un discurso discontinuo, bajo el que no subyace un meta-discurso que sustente todos los discursos posibles (Foucault, 2008).

6. La introducción de un problema de incapacidad explicativa completa en la sociología, puede indicar varias cosas distintas a puntualizar: A) La evasión de lo real/social frente a cualquier potencial explicación, lo que es lo mismo, la inconmensurabilidad objetiva de lo social, porque: a) tal cosa sea en definitiva imposible, señalando una opacidad óntica de lo social; b) una causa inherente a lo real/social imposibilite tal cosa, indicando o condicionando una fisura óntica entre realidad y conocimiento (cosa que no puede seguirse de ningún argumento, incluso metafísico, en tanto, destruiría toda posibilidad del conocimiento, socavando la categoría clásica de sujeto y la mucho más contemporánea de agencia); y, c) no se ha dado un suficiente desarrollo del conocimiento científico que permita tal cosa. La opción (A: 6Ac) carga el peligro de una hipótesis radicalmente progresista (evolucionismo fuerte). B) Carencias de la estructura cognitiva humana que imposibilitan la comprensión completa de la realidad social, debido a una limitación cognoscitiva. C) Limitaciones del proceso de construcción del conocimiento actual en la medida que solo responda a una pretendida naturaleza humana. Desde el punto de vista de las ciencias sociales, se ha llegado a la constatación de que el proceso de conocimiento no se realiza por la simple participación de sujetos individuales (ni siquiera bajo el concepto de descubrimiento en el marco de la ciencia extraordinaria definida por Kuhn u otros enfoques más radicales al respecto, caso del modelo anarquista de Feyerabend $-\underline{1986}-$, o del principio del actor-red propio de la sociología simétrica, sino por una institucionalidad cultural inherente y amplia de la ciencia, y de la condición humana que hace intrínseco lo individual/colectivo y la propensión al conocimiento; explicación que podría llevar, a la argumentación respecto de un desarrollo epistemológico fuerte indicada en (A: $\mathbf{A c}$ ); rechazable si se define desarrollo como condición de posibilidad. Esta situación (A: 6C) parece poco probable, dado el mismo debate filosófico previo, mismo que en ninguna forma se puede calificar de simplemente especulativo. D) La complejidad general de lo real/social que, siguiendo el anterior aspecto, supone, la imposibilidad de una reducción cognitiva unívoca, por un lado y, por otro, el requerimiento de múltiples enfoques y/o posiciones de observación que permitan el conocimiento de lo social, e incluso del mundo objetivo natural.

7. Las posturas de continuidad y discontinuidad absolutas, parecen altamente refutables, en tanto socavarían los principios tomados hipotéticamente de causalidad, condicionamiento relativo, unidad y totalidad, pese a: A) La irreductibilidad a leyes estables de las condiciones de la realidad social, salvo (A: 4Da-4Dd). B) La definición de las leyes sociales a prelegalidades que actúan como factores relativos de condicionamiento. C) La sujeción parcial de 
las leyes sociales a criterios de discernimiento, tanto en un plano puramente ideacional probable como tecnológico/teleológico incluyendo la justificación del criterio de libertad (Giménez, 2010). D) La fuga inmediata de lo real/social respecto de toda aproximación epistemológica mediata o una explicación evolutiva fuerte. E) La reducción del fenómeno humano a una orientación sociocognitiva (que contiene uno de los factores fundamentales de la constitución social: la imaginación).

8. El problema de la forma pasiva de la utopística, dentro de la ciencia en general, se ha enredado en las siguientes consecuencias: A) Positivización empiricista de las ciencias naturales, con un debate a posteriori (tecno-científico) de sus efectos, sin que se dé una visualización del carácter social a priori de estas mismas ciencias, ante todo bajo la diferenciación clásica entre los denominados contextos de justificación y de descubrimiento (Domènech y Tirado, 1998,16). B) Positivización gnoseológica empiricista de las ciencias sociales, trasladando el debate ético a la forma activa de la utopística.

9. A los eventos técnicos propios de la sociología (conscientes o no conscientes, residuales o no residuales, eticopolíticamente normativos o no normativos), se les puede denominar sociotécnicas, cuyas características serían: A) Establecen un distinción $\mathbf{T} /[\mathbf{c}(\mathbf{s} \rightarrow \mathbf{0})]$, donde: a) c corresponde a un específico proceso de conocimiento $(\mathbf{s} \rightarrow \mathbf{0})$; b) / indica una ruptura y, a la vez, una transformación mediada por $[\mathbf{c}(\mathbf{s} \rightarrow \mathbf{0})]$ de la realidad de carácter residual, mediante la que el conocimiento deviene como una función probable y general de saber (no necesariamente de conocimiento); c) T implica una transformación de o en $\mathbf{o}^{\prime}$, de $\mathbf{s}$ en $\mathbf{s}^{\prime}$ y de $\mathbf{c}$ en $\mathbf{c}^{\prime}$, producto de la ruptura hiancial /; y, d) T no incluye una necesaria visualización reflexiva del conocimiento como (...). Por ahora, no se debate si c, o, y / corresponden a una misma imbricación óntica. B) Toda sociotécnica supone un cambio de toda o una parte de la estructura óntica de la realidad socio-natural en general, no necesariamente en particular, lo que introduce una recursividad subtrascendental (dentro del tiempo imaginario individual/social) o estrictamente trascendental (en el tiempo entrópico) esperada o inesperada bajo la forma cancelada de $\mathrm{T} /[\mathbf{c}(\mathbf{s} \rightarrow \mathbf{0})]$.

10. El problema se hace patente cuando no se puede establecer, de manera segura, precisa ni inmediata, la naturaleza del cambio en dicha estructura: $\mathbf{T}$ se transmuta en $\mathbf{T}^{\prime}$ rebasando todos los factores participantes. La distancia espontánea o no controlada entre $\mathbf{T}$ y $\mathbf{T}^{\prime}$ ( $\sin$ mediación tecnológica ( $\left.\mathbf{T}^{\prime \prime} / \mathbf{c}^{\prime \prime}\left[\mathbf{T}^{\prime} /\left[\mathbf{c}^{\prime}(\mathbf{s} \rightarrow \mathbf{0})\right]^{\prime}\right]^{\prime \prime}\right)$ puede denominarse evento técnico instrumental (ETI), el cual está cargado de transformaciones realidad/saber (sobre las que no siempre operan posteriores actos de conocimiento (...)) y que pueden ser: a) esperados (ETE); b) no esperados (ETNI); c) no calculables (ETNC); y, d) riesgo (ETR).

11. Se entiende por evento objetivo todo evento circunscrito a la propia condición/evolución de la naturaleza objetiva en su conjunto. No existen eventos fuera de la evolución de la naturaleza (dicho de manera radical nada puede actuar, más allá de/fuera de la naturaleza). Si existiesen tales eventos exógenos, simplemente indicarían la existencia de un mayor alcance óntico. Por lo que: el conocimiento constituye evento de la naturaleza (se discrepa, por supuesto, de la postura realista y objetivista extrema de Mario Bunge). 
12. Todo evento de conocimiento, epistémicamente justificable, requiere por ello, de un posterior evento de conocimiento (reflexivo) que identifique su especificidad real/social (ciencia de la ciencia). Todo evento de conocimiento que no se sujete a este criterio es evento salvaje de conocimiento (= saber) que, potencialmente, supone un evento riesgo óntico negativo (una transformación de la realidad socio/natural con consecuencias inesperadas; con un potencial de daño de la vida, siempre superior a cero).

13. La salida al conflicto tecnología/destrucción ecosistémica camina entonces por el lado de la ética: más que de una ética de la investigación, se requiere de una ética de los límites de la ciencia como hecho socio/natural, de sus efectos real/objetivos activos y pasivos, técnico/cognoscitivos, evidentes y residuales, lo cual implica: La necesidad permanente de reflexión sobre el papel y efectos de la sociología, planteando límites cognoscitivos y tecnológicos conscientemente electos, por lo menos mientras no queden claros dichos efectos realidad.

14. Resulta conveniente indicar algunos supuestos ya anunciados o agregar algunos supuestos más a los que ya se ha venido señalando en las problematizaciones previas: A) Irreductividad estructural, sistémica o funcional de los movimientos sociocognitivos. De ahí que se recurra criterios amplios al definir a las denominadas sociedades o lo que algunos autores denominan sociedades parciales: a) Comunidades; b) formaciones sociales; c) configuraciones sociales; d) sistemas; e) conjuntos de acciones diferenciadas en el caso de las teorías de la acción (Gutiérrez, 2000; Iglesias y Herrara, 2005); f) multiplicación de los juegos en el caso de las teorías de juegos; cadenas de actor-red (sociología simétrica); así como a la figura categorial densa de sociocognición. B) Independencia respecto de la categoría de sujeto de las operaciones, las acciones y movimientos sociocognitivos, con una consecuente proliferación, diferenciación y reconstitución de las agencias (más allá de las figuras clásicas de conciencia, sujeto, individuo, persona), producto, a su vez, de: a) una proliferación plural de las agencias sociocognitivas; b) una desregulación de los agencias clásicas debido a la introducción de nuevas variables ligadas con la cultura y el lenguaje (Arce, 2007: pp. 169-191). C) Autoorganización relativa de los procesos social/objetivos, con independencia también relativa de la sociocognición moderna clásica (subjetivista). D) Diferenciación creciente de los procesos social/objetivos en subprocesos también de naturaleza multidimensional. E) Pérdida de la determinación histórica exclusiva a las operaciones mnésico/entrópicas presente/futuro, a favor de orientaciones determinativas y no determinativas mutidimensionales, provenientes y/o garantizadas desde el tiempo imaginario. En relación con esto, discontinuidad óntica bajo el tiempo entrópico. F) Multiplicación multidimensional de las agencias y las orientaciones de la acción: a) ruptura de la orientación determinada solo por condiciones del espaciotiempo entrópico a favor también de diversas formas de movimiento espaciotemporales imaginarias o virtuales (supone una densificación multidimensional del espacio-tiempo; también sujeta a la entropía); b) multiplicación cuantitativa, aunque no necesariamente cualitativa, de los soportes tecnomateriales de la realidad socio/objetiva; c) multiplicación de los mundos y recursos imaginarios, producto de la creciente densificación del evento sociocultural. Es decir, si $(\mathbf{t})$ = tiempo entrópico, $(\mathbf{e})$ = espacio e $(\mathbf{i})$ = espacio-tiempo imaginario, por lo tanto: $\left[\mathbf{c}^{\infty}\left(\mathbf{c}^{\prime \prime}\left(\mathbf{c}^{\prime}(\mathbf{c}(\mathbf{s} \rightarrow \mathbf{0}))^{\prime}\right)^{\prime \prime}\right) \infty\right]^{[\mathrm{e} / t / \mathrm{i}]} ; 0$ bien: $(\ldots)^{[\mathrm{e} / \mathrm{t} / \mathrm{i}]}$. Las relaciones entre las dimensiones 
espaciotemporales entrópicas e imaginarias se intersecan y comunican produciendo efectos real/objetivos mediante procesos sociales interdimensionales. 